\title{
"An optimized Distributed Video Coding using Turbo Codes and Zero Motion Skip Encoder Strategy"
}

\author{
Chitra.M $^{1}$, Roopa.M. ${ }^{2}$ \\ ${ }^{l}$ pursuing M.Tech Degree under V.T.U at Dayananda Sagar College of Engineering \\ ${ }^{2}$ Assistant Professor in the Department of Electronics and communication, Dayananda sagar college of \\ engineering, Bangalore, INDIA
}

\begin{abstract}
Distributed coding enables higher compression rate of a video signal. Till date we used a complex encoder and simple decoder. The advantage of having a simpler encoder and a complex decoder is increase in the compression ratio. Here in this project an optimized distributed video coding scheme is proposed which is a combination of an intraframe encoder and an interframe decoder. The distributed video coding scheme is based on punctured turbo codes. Turbo codes are punctured to generate scalable compressed video bit streams. An improved turbo coding performance leads to an overall performance improvement. Zero motion skip encoding strategy is used so that the decoder is made aware of these zero motion blocks such that encoding of these blocks can be avoided. The decoder may use this to improve the performance.
\end{abstract}

Keywords: Distributed video coding, turbo codes, zero motion skip encoder strategy.

\section{INTRODUCTION}

In traditional video coding schemes, asymmetric complexity exits in encoder and decoder. The motion estimation modules dominating the encoding complexity are usually very time-consuming. However, in many applications such as sensor networks and multi-camera scenarios, the complex compression has to be done in the processors with low processing capabilities. In this case, the encoding complexity becomes a big burden. As we know, most of the encoding complexity comes from the correlation exploration modules such as motion estimation.

The distributed video coding scheme is based on punctured turbo codes. Turbo codes use three simple ideas: parallel concentration of codes to allow simpler decoding; interleaving to provide better weight distribution; and soft decoding to enhance decoder decisions and maximize the gain from decoder interaction. Turbo codes are punctured to generate scalable compressed video bit streams. An improved turbo coding performance leads to an overall performance improvement.

Zero motion skip encoding strategy is used so that the decoder is made aware of these zero motion blocks such that encoding of these blocks can be avoided. The decoder may use this to improve the performance.

\section{DISTRIBUTED VIDEO CODING}

Distributed video coding schemes have been proposed to provide potential reverse in computational complexity for decoder and encoder. The theoretical background of these schemes is based on Slepian-Wolf and Wyner-Ziv distributed video coding theories. The distributed video coding schemes proposed recently have a distributed video coding schemes proposed have general architecture: intra encoding and inter decoding. At the encoder, error control codes are applied to each frame to generate syndrome bits.

Distributed coding is a new paradigm for video compression, based on Slepian and Wolf's and Wyner and Ziv's information-theoretic results from the 1970s. This paper reviews the recent development of practical distributed video coding schemes. Wyner-Ziv coding, i.e., lossy compression with receiver side information, enables low-complexity video encoding where the bulk of the computation is shifted to the decoder. Since the interframe dependence of the video sequence is exploited only at the decoder, an intraframe encoder can be combined with an interframe decoder [1].

The rate-distortion performance is superior to conventional intraframe coding, but there is still a gap relative to conventional motion-compensated interframe coding. Wyner-Ziv coding is naturally robust against transmission errors and can be used for joint source-channel coding. A Wyner- Ziv MPEG encoder that protects the video waveform rather than the compressed bit-stream achieves graceful degradation under deteriorating channel conditions without a layered signal representation [4][5]. 
Distributed compression refers to the coding of two (or more) dependent random sequences, but with the special twist that a separate encoder is used for each. Each encoder sends a separate bit-stream to a single decoder which may operate jointly on all incoming bitstreams and thus exploit the statistical dependencies. The simplest system that we have investigated is the combination of a pixel-domain intraframe encoder and interframe decoder system for video compression.

\section{THE PROPOSED VIDEO CODING ARCHITECTURE}

A subset of frames, regularly spaced in the sequence, serve as key frames, $K$, which are encoded and decoded using a conventional intraframe $8 \times 8$ Discrete Cosine Transform (DCT) codec. The frames between the key frames are "Wyner-Ziv frames" which are intraframe-encoded but interframedecoded. For a Wyner-Ziv frame, $S$, each pixel value is uniformly quantized with $2 M$ intervals. We usually incorporate subtractive dithering to avoid contouring and improve the subjective quality of the reconstructed image [1].

A sufficiently large block of quantizer indices $q$ is provided to the Slepian-Wolf and the rest of the frames are Wyner-Ziv frames. The side information is generated by motion-compensated interpolation using adjacent reconstructed frames. The plots show the rate and PSNR, averaging over both key frames and WynerZiv frames. We compare our results to (i) DCT-based intraframe coding (all frames are encoded as I frames) and (ii) H.263+ interframe coding with an I-B-B-B-I predictive structure. As it can be seen from the plots, the pixeldomain Wyner-Ziv coder performs 2 to $5 \mathrm{~dB}$ better than conventional intraframe coding. There is still a significant gap towards H.263+ interframe coding.

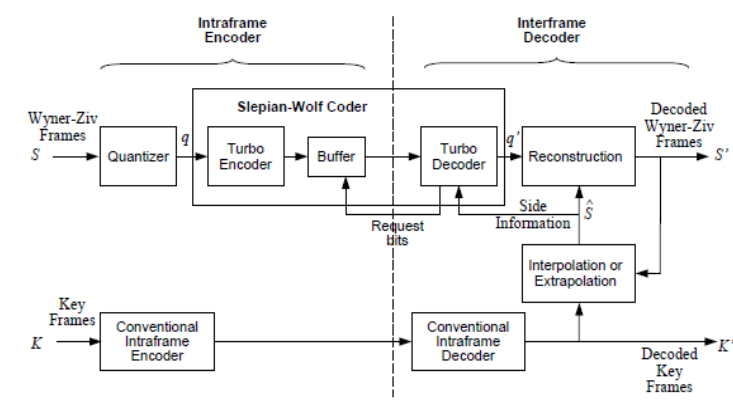

Fig 1: Architecture of WZ codec

The WZ codec is shown in Fig. 1. The WZ codec is used to encode and decode only the even frames. Each pixel $u$ of $F 2 i$ is quantized using a uniform quantizer with $2 M$ levels to produce a quantized symbol q.2 The quantized symbol $\mathbf{q}$ is then converted to a binary codeword [ $q 0 \ldots q M-1]$. A sufficient number of symbols are collected and are encoded using a turbo code. The turbo encoding is done as follows. The quantized symbols are converted to binary codewords and encoded using a recursive systematic convolutional (RSC) code to produce a systematic bit stream and a parity bit stream. The quantized symbols are interleaved and then encoded using another identical RSC code to produce a second parity bit stream. The interleaving is performed on the pixels, although this is not strictly required based on the way that the decoder is implemented. The decoder has SI $(S 2 i)$ about the current frame $F 2 i$. In the video coding literature, the difference between the pixels of $F 2 i$ and $S 2 i$ is usually modeled as a Laplacian random variable,

$$
\left.\left\{S_{2 t}\right\}_{m, n}=\left\{F_{2 t}\right\}_{m . n}+\eta \ldots i\right)
$$

where $(m, n)$ indexes the pixel location and $\eta$ is a Laplacian random variable with density function $p(\eta)=\alpha 2$ $\mathrm{e}^{-} \alpha|\mathrm{n}|$. Thus, S2i can be considered to be the output of a channel with Laplacian noise to which the original frame F2i is the input. This fictitious channel that arises because of the correlation between the frames of the video is sometimes referred to as the correlation channel. Since a noisy version of F2i is available at the decoder in the form of S2i, the systematic part of the turbo code need not be transmitted. It is shown in Fig. 1 that the systematic part is discarded. The parity streams generated by the two RSCs can be punctured to obtain any desired rate. Compression is achieved when the number parity bits transmitted is smaller than the number of bits input to the turbo encoder. The decoder in the receiver has to estimate the original frame F2i from the SI S2i and 
the parity bits from the turbo code. The parity bits are assumed to be transmitted through an error-free channel. This is a common assumption in source coding, wherein the compressed bits are assumed to be error-free at the source decoder. The WZ video decoder consists of a turbo decoder followed by a reconstruction function. The WZ decoder estimates the pixels of the original frame (F2i) in a two-step process. First, the turbo decoder operates on the transmitted parity bits along with the side-information to produce an estimate of the quantized symbols, q1. This estimate $\mathbf{q 1}$ and the SI S2i are used to reconstruct an estimate u1 of the original pixel u in frame F2i [2]. The reconstruction function used is given by,

$$
\begin{aligned}
\mathrm{u} 1=\mathrm{E}\left(\mathrm{u} / \mathrm{q} 1-\mathrm{S}_{2 \mathrm{t}}\right)= & \mathrm{b}_{1}, \quad \mathrm{v} \leq \mathrm{b}_{\mathrm{t}} \\
& \mathrm{v}, \quad \mathrm{b}_{\mathrm{t}}<\mathrm{v}<\mathrm{bu} \\
& \mathrm{b}_{\mathrm{u}}, \quad \mathrm{v} \geq \mathrm{b}_{\mathrm{u}}
\end{aligned}
$$

Where $\mathrm{v}$ is the pixel in S2i that forms the side-information for $\mathrm{u}$, and $\mathrm{b}_{1}$ and $\mathrm{b}_{\mathrm{u}}$ are the lower and upper boundaries of the quantization bin indexed by q1. That is, if a pixel has a value s such that $\mathrm{bl} \leq \mathrm{s}<\mathrm{bu}$, then the quantized symbol representing $\mathrm{s}$ is q1. Thus, if the side-information $\mathrm{v}$ lies in the reconstruction bin indexed by $\mathbf{q} 1$, then $\mathrm{u} 1$ takes on the value of $\mathrm{v}$. If $\mathrm{v}$ lies outside the reconstruction bit of q1, then $\mathrm{u} 1$ takes on the value of the boundary closest to $\mathrm{v}$.

\section{EXPERIMENTAL RESULTS}

To test the performance of the proposed improved Turbo codes for distributed source coding, 10000 randomly generated binary sequences are used. The length of each sequence is 1024 bits and the 1,0 s are uniform distributed. After Turbo encoding, a certain number of bits of systematic bits are selected randomly to change the sign. The resulted bits streams are into Turbo decoders [6]. Fig 2 gives the decoding bit error rate performances of Turbo codes at code rate 2/3; Fig 3. gives the decoding bit error rate performances at code rate 4/5. From the results we can see the decoding BER performances improved significantly by using proposed improved Turbo codes for distributed source coding [9][10].

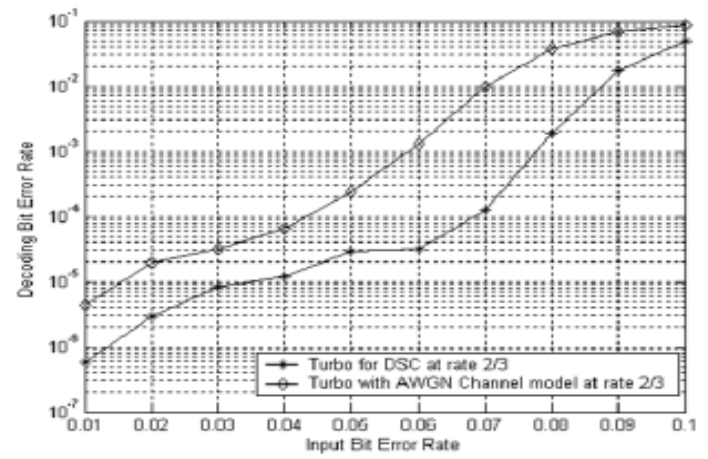

Fig 2: Decoding BER performance for 2/3 Turbo codes

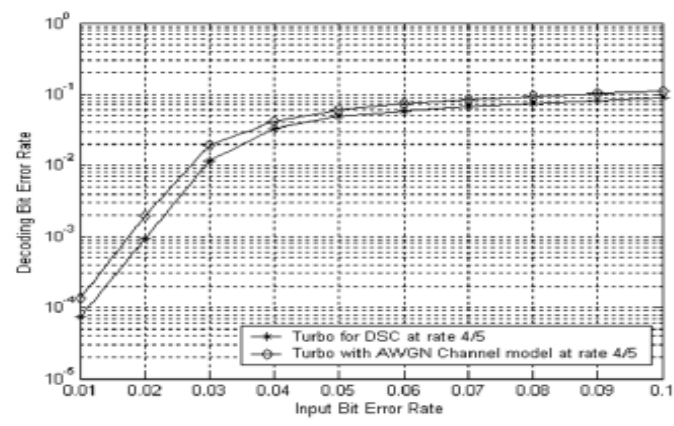

Fig 3: Decoding BER performance for $4 / 5$ Turbo codes www.iosrjournals.org 
The proposed architecture is been tested with a video of 5 minutes video and various parameters have been compared. The encoding and decoding was carried out by converting each spixel of the frames of the video to its intensity levels. Later these intensity levels were truncated by certain threshold level.The encoding and decoding of these levels are carried out using a special Turbo coding method. The results are compared with various iteration levels of Turbo codes, with and without puncturing Turbo codes, different seed values to the decoder.

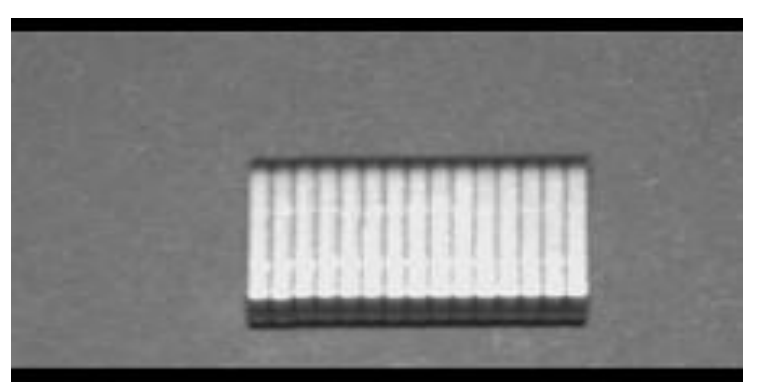

Fig 4: A frame of the actual video clip

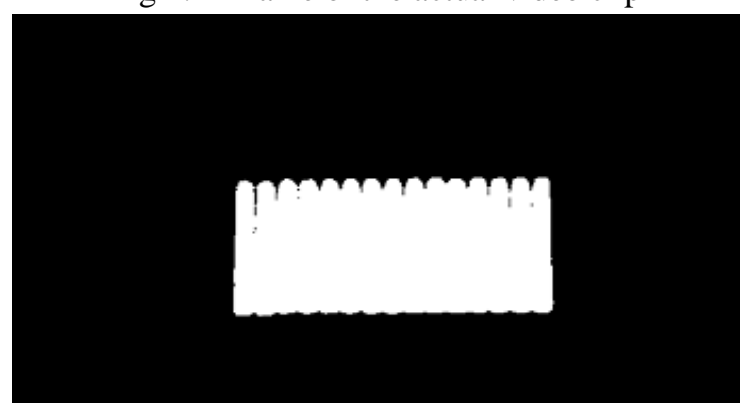

Fig 5: The intensity levels of the frame of actual video clip

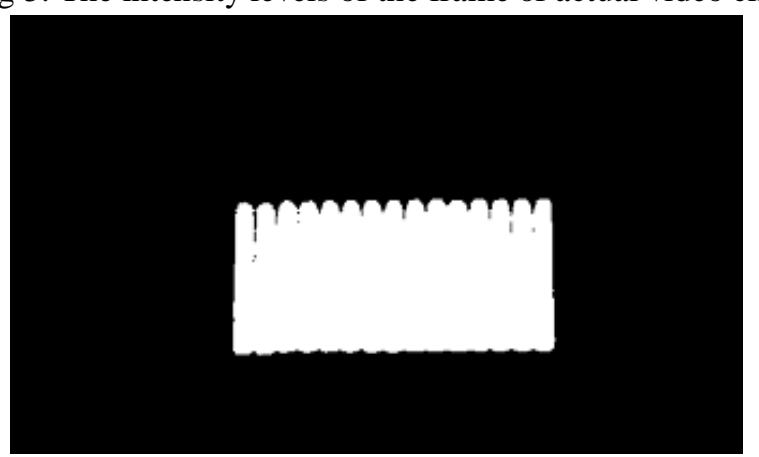

Fig 6: The decoder frame of the video clip (only intensity levels)

\section{CONCLUSION}

In this paper, we reported the investigation about the behaviour of turbo coding with puncturing. A low punctured turbo codes and zero motion skip encoding strategy for distributed video coding was presented. Using the zero motion vector blocks, the generation of side information and the design of the punctured turbo codes can be integrated to improve the overall coding performance. Low level of encoder complexity is proposed. No motion estimation or compensation is required. The proposed architecture gives a better performance than conventional intraframe coding at low complexity. 


\section{REFERENCES}

[1] Q. Zhu and Z. Xiong, "Layered Wyner-Ziv video coding," Submitted to IEEE Transactions on Image Processing, July 2004.

[2] J. Cardinal and G. V. Asche, "Joint entropy-constrained multiterminal quantization," in Proc. IEEE International Symposium on Information Theory (ISIT), Lausanne, Switzerland, June 2002, p. 63.

[3] Z. Xiong, A. Liveris, and S. Cheng, "Distributed source coding for sensor networks," IEEE Signal Processing Magazine, vol. 21, no. 5, pp. 80-94, Sept. 2004.

[4] S. S. Pradhan, J. Kusuma, and K. Ramchandran, "Distributed compression in a dense microsensor network," IEEE Signal Processing Magazine, vol. 19, no. 2, pp. 51-60, Mar. 2002.

[5] J. Kusuma, L. Doherty, and K. Ramchandran, "Distributed compression for sensor networks," in Proc. IEEE International Conference on Image Processing (ICIP), vol. 1, Thessaloniki, Greece, Oct. 2001, pp. 82-85.

[6] A. Aaron and B. Girod, "Compression with side information using turbo codes," in Proc. IEEE Data Compression Conference, Snowbird, UT, Apr. 2002, pp. 252-261.

[7] S. S. Pradhan, J. Kusuma, and K. Ramchandran, "Distributed compression in a dense microsensor network," IEEE Signal ProcessingMagazine, vol. 19, no. 2, pp. 51-60, Mar. 2002.

[8] S. S. Pradhan and K. Ramchandran, "Distributed source coding using syndromes (DISCUS): Design and construction," in Proc. IEEE Data Compression Conference, Snowbird, UT, Mar. 1999, pp. $158-167$.

[9] J. D. Slepian and J. K. Wolf, "Noiseless coding of correlated information sources," IEEE Transactions on Information Theory, vol. IT-19, pp. 471- 480, July 1973.

[10] "Distributed source coding: Symmetric rates and applications to sensor networks," in Proc. IEEE Data Compression Conference, Snowbird, UT, Mar. 2000, pp. $363-372$.

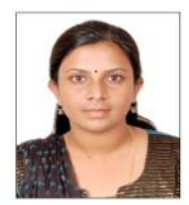

Chitra.M. received B.E, Degree from V.T.U. in 2007, worked as a lecturer in Acharya Patashala College of Engineering for three years. Currently pursuing M.Tech Degree under V.T.U at Dayananda Sagar College of Engineering.

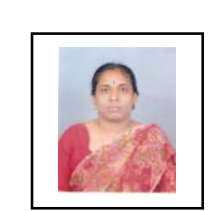

Roopa. M. received B.E, Degree from U.V.C.E Bangalore University, Bangalore In 1990, M.E, Degree from U.V.C.E, Bangalore University, Bangalore, India in 1994, pursuing Ph.D in the Department of Applied Electronics, Gulbarga University, Gulbarga, INDIA, currently she is working as Assistant Professor in the Department of Electronics and communication, Dayananda sagar college of engineering, Bangalore, INDIA, her research interests include Design of Digital circuits, VLSI Design and Embedded systems. 